\title{
Prevenção e fatores relacionados à ideação suicida em adolescentes nas entrelinhas de uma revisão narrativa
}

\author{
Prevention and factors related to suicidal ideation in adolescents between the lines of a \\ narrative review
}
Prevención y factores relacionados con la ideación suicida en adolescentes entre líneas de una revisión narrativa

Luísa Vieira Santos ${ }^{1 *}$, Bárbara Camargo Levy², Bruna Damis Resende ${ }^{3}$, Caroline Silva de Araujo Lima ${ }^{4}$ ,Danielle Raphaela Alves ${ }^{5}$, Geovanna Siqueira Santana ${ }^{6}$, Maria Letícia de Oliveira Ruela ${ }^{7}$, Jéssica Milanêz Melo de Araújo ${ }^{8}$, Thayna de Andrade Romeu Alexandre ${ }^{9}$, Eduarda Faria Abrahão Machado ${ }^{10}$.

\section{RESUMO}

Objetivo: O presente artigo realizou uma revisão acerca dos fatores associados e estratégias de prevenção sobre suicídio e depressão em adolescentes. Revisão bibliográfica: Sabe-se que o período da adolescência é um momento de formação da identidade do jovem para a vida adulta e que, para isso, é necessário um ambiente seguro e acolhedor. Infelizmente, este momento é marcado por uma maior vulnerabilidade, visto que as mudanças e adaptações psicossociais, desta fase, tem uma grande influência sociocultural, através das mídias sociais, dos padrões impostos diariamente pela sociedade e pela família. A ideação e o comportamento suicida não são considerados doenças, mas apresentam forte relação com a presença de alguns transtornos mentais, como o transtorno depressivo, os transtornos por uso de substâncias, a esquizofrenia e alguns transtornos de personalidade. Além da relação com a desigualdade social, o desemprego, a instabilidade familiar, dentre outros fatores sociais. Considerações finais: Esse trabalho verificou o aumento de casos de suicídio e depressão em adolescentes e suas relações multifatoriais, tendo como objetivo investigar os fatores de risco associados e as estratégias de prevenção, a fim de auxiliar pais e profissionais da saúde a compreenderem o sofrimento psicológico e promoverem a saúde mental a esses adolescentes.

Palavras-chave: Suicídio, Adolescência, Fatores de risco.

\footnotetext{
ABSTRACT

Objective: This article conducted a review of the associated factors and prevention strategies for suicide and depression in adolescents. Bibliographic review: It is known that the period of adolescence is a time of identity formation of young people for adulthood and that, for this, a safe and welcoming environment is necessary. Unfortunately, this moment is marked by a greater vulnerability, since the psychosocial changes and adaptations of this phase have a great sociocultural influence, through social media, and the standards

${ }^{1}$ Centro Universitário de Belo Horizonte (UNIBH), Belo Horizonte - MG. *E-mail: vieiraluisa4@gmail.com

2 Universidade Cidade de São Paulo (UNICID), São Paulo - SP.

${ }^{3}$ Faculdade Faceres, São José do Rio Preto - SP.

${ }^{4}$ Faculdade Dinâmica do Vale do Piranga (FADIP), Ponte Nova - MG.

${ }^{5}$ Centro Universitário do Espírito Santo (UNESC), Colatina - ES.

${ }^{6}$ Universidade de Gurupi (UNIRG), Gurupi - TO.

${ }^{7}$ Faculdade Ciências Médicas de Minas Gerais (FCMMG), Belo Horizonte - MG.

${ }^{8}$ Centro Universitário do Planalto Central Apparecido dos Santos (UNICEPLAC), Gama - DF.

${ }^{9}$ Centro Universitário Presidente Antônio Carlos (UNIPAC), Juiz de Fora - MG.

10 Faculdade de Medicina de Jundiaí, Jundiaí - SP.
} 
imposed daily by society and the family. Suicidal ideation and behavior are not considered illnesses, but they are strongly related to the presence of some mental disorders, such as depressive disorder, substance use disorders, schizophrenia, and some personality disorders. Besides the relationship with social inequality, unemployment, family instability, among other social factors. Final considerations: This study verified the increase of suicide and depression cases in adolescents and their multifactorial relations, aiming to investigate the associated risk factors and prevention strategies in order to help parents and health professionals understand the psychological suffering and promote mental health to these adolescents.

Keywords: Suicide, Adolescent, Risk factors.

\section{RESUMEN}

Objetivo: El presente artículo realiza una revisión sobre los factores asociados y las estrategias de prevención del suicidio y la depresión en adolescentes. Revisión bibliográfica: Se sabe que el período de la adolescencia es un momento de formación de la identidad del joven para la vida adulta y que, para ello, es necesario un entorno seguro y acogedor. Desgraciadamente, este momento está marcado por una mayor vulnerabilidad, ya que los cambios y adaptaciones psicosociales de esta fase tienen una gran influencia sociocultural, a través de los medios de comunicación social, las normas impuestas diariamente por la sociedad y la familia. La ideación y el comportamiento suicida no se consideran enfermedades, pero están muy relacionados con la presencia de algunos trastornos mentales, como el trastorno depresivo, los trastornos por consumo de sustancias, la esquizofrenia y algunos trastornos de la personalidad. Además de la relación con la desigualdad social, el desempleo, la inestabilidad familiar, entre otros factores sociales. Consideraciones finales: Este estudio verificó el aumento de los casos de suicidio y depresión entre los adolescentes y sus relaciones multifactoriales, con el objetivo de investigar los factores de riesgo asociados y las estrategias de prevención para ayudar a los padres y a los profesionales de la salud a comprender el sufrimiento psicológico y promover la salud mental de estos adolescentes.

Palabras clave: Suicidio, Adolescencia, Factores de riesgo.

\section{INTRODUÇÃO}

A adolescência é um período do desenvolvimento humano, que se caracteriza pela transição entre a infância e a idade adulta e todas as transformações sociais existentes. Essa fase também corresponde a alterações biológicas do sistema nervoso central que podem facilitar comportamentos inconstantes, o que pode gerar relações conflituosas com os responsáveis. Todas as transformações biopsicossociais dessa fase podem conduzir os adolescentes a quadros de depressão, culminando em ideações suicidas e ao ato em si (CIDADE NOP e ZORNIG SMAJ, 2021).

A depressão é uma doença extremamente grave, caracterizando-se como um problema de saúde pública mundial. De acordo com a Organização Mundial de Saúde (OMS), o suicídio é a segunda causa de morte mais comum entre jovens de 15 a 29 anos, sendo que a quantidade de óbitos gira em torno de 800.000 por ano no mundo e 12.000 por ano no Brasil e estima-se que para cada indivíduo que cometa suicídio, 29 outros farão ao menos uma tentativa (OLIVEIRA JWT, et al., 2020).

Dessa forma, o comportamento suicida na adolescência é desencadeado por diferentes causas, normalmente, relacionadas aos conflitos internos, sentimentos depressivos e ansiedade que acontecem devido às funções sociais que ainda não estão bem estabelecidas nessa fase, o que causa sentimento de insuficiência e tristeza profunda (DURKHEIM E., 2011). Nesse sentido, de acordo com o Ministério da Saúde (MS), a maior parte das vítimas apresenta algum distúrbio psíquico associado, sendo a depressão o mais frequente (MINISTÉRIO DA SAÚDE, 2019).

Segundo o estudo de Ribeiro JM e Moreira MR (2018), de 2014 a 2018, a maior parte de mortalidade por autoextermínio foram de indivíduos do sexo masculino, negros e com idade entre 10 e 29 anos. A predileção 
por gênero se deve a maior exposição a alguns fatores de risco associados ao gênero masculino, como maior agressividade, impulsividade e drogadição. Dentre os fatores de risco, pode-se destacar os socioeconômicos, como desigualdade e desemprego, sendo que quanto maiores as taxas, maiores os índices de suicídio local (MINISTÉRIO DA SAÚDE, 2019; JAEN-VARAS D, et al., 2019).

Existem formas de se avaliar o risco de suicídio na avaliação clínica. Uma dessas escalas, chamada de Escala de Ideação Suicida de Beck (BSI), é composta por 21 itens que podem ser pontuados de 0 a 3 sendo capaz de detectar a ideação suicida e o tamanho da motivação pessoal para a ação em si, quanto maior a pontuação maior o risco de ideação suicida e de chance de tentativa. Essa escala pode ser um instrumento útil nas avaliações desses adolescentes. Em um estudo transversal, $48,71 \%$ dos adolescentes pesquisados apresentaram ideação suicida e isso pode ser explicado em função da pressão psicológica vivenciada nessa fase, bem como a sensação de solidão, a ausência dos pais, amigos, o uso de álcool e outras drogas (BIRMAN J, 2016).

Este estudo justifica-se pelo aumento crescente nas taxas de suicídio dos adolescentes, visto que esse é um período particularmente vulnerável a esse tipo de acontecimento em razão das transformações inerentes a essa fase, a fim de auxiliar pais e profissionais da saúde a compreenderem o sofrimento psicológico e garantir a saúde mental dos adolescentes. Por isso, define-se como objetivo do presente estudo investigar os fatores de risco associados ao problema e estratégias de prevenção associados à ideação suicida em pacientes adolescentes.

\section{REVISÃO BIBLIOGRÁFICA}

\section{Epidemiologia}

A tentativa de suicido não é ocasionada tendo em vista uma causa única ou um evento isolado, mas sim como um fenômeno multifatorial. Entre $2 \%$ e $12 \%$ dos jovens do mundo tentaram suicídio e, atualmente, isso é comumente um motivo de consulta em saúde mental e um problema de saúde pública muito discutido. $O$ estudo de Caballero MA (2017) mostrou que o suicídio é a terceira causa de morte entre adolescentes e que as taxas globais de suicídio, nos últimos 45 anos, alavancaram cerca de 60\%. De acordo com Serrano-Ruiz CP e Olave-Chave JA (2017), mais de 800.000 pessoas se matam a cada ano. Além disso, estima-se que cada pessoa que comete esse ato já fez tentativas anteriores. Suicídios podem ocorrer em qualquer idade, mas é a segunda causa de morte entre 15 e 29 anos (TSAI AC, et al., 2015).

O Brasil é um país com o Produto Interno Bruto (PIB) consideravelmente alto, porém com os níveis de desigualdade social e econômica também muito altos, o que implica diretamente na saúde da população de quase 200 milhões de habitantes, manifestando-se como uma causa verídica do suicídio. O imenso território com diferenças culturais e socioeconômicas demonstra a variação das taxas de suicídio. Entre 1997 e 2015, foi descrito um número elevado de suicídio entre jovens e adultos e isso torna perceptível a qualidade de vida calamitosa da população. Cerca de $85 \%$ dos suicídios ocorreram na população jovem e adulta (15-59 anos), e as taxas médias de suicídio em homens foram maiores do que em mulheres, independente da faixa etária ou do estado brasileiro (TSAI AC, et al., 2015).

Tanto as tentativas de suicidio quanto o ato consumado têm apresentado taxas crescentes entre os jovens. Nesse sentido, $98 \%$ dos suicidas apresentam certas barreiras psicológicas ao se suicidar, principalmente as emocionais. Além disso, mais de $70 \%$ das crianças e adolescentes com transtornos de humor graves não são diagnosticados ou tratados adequadamente (CABALLERO MA, et al., 2017).

\section{Causas}

O suicídio é uma das principais causas de morte entre adolescentes e jovens em todo o mundo. Os processos mentais associados ao suicídio são vivências depressivas intensas, ódio, raiva, ansiedade, associadas a uma autoimagem desqualificada. No entanto, o suicídio envolve extrema dor mental, onde a morte é vista como única solução para cessá-lo (JAEN-VARA D, et al., 2019; SERRANO-RUIZ CP e OLAVECHAVES JA, 2017). 
$\mathrm{Na}$ fase da adolescência o enfrentamento com as atribuições, afazeres, cobranças vivenciadas nessa fase, impostos em sua maior parte pela família, e também pela sociedade e mídia social, que associado à imaturidade e inexperiência, tendem a causar angústia, apreensão e aflições que quando não bem abordado propiciam ideação, planejamento e a tentativa de autoextermínio (CABALLERO MA, et al).

Compreensivelmente, grande parte desses comportamentos está relacionada a transtornos mentais. Os mais comuns são depressão, esquizofrenia e psicose. O abuso de drogas e de álcool também tem um impacto considerável na ideação suicida. A depressão e outras psicopatologias são causas muito importantes, como desespero, perda de interesse nas atividades diárias e tristeza, levando as pessoas a pensar que a autodestruição será um alívio. Outro motivo relacionado aos adolescentes é a desilusão amorosa, principalmente as mulheres, pois algumas pessoas atribuem grande importância às relações amorosas (SILVA LLT, et al., 2015).

É importante ressaltar que, em alguns casos, não é fácil identificar com precisão os indícios que podem desencadear o aumento do suicídio entre os jovens. Na perspectiva dos profissionais da área, pode-se dizer que a influência das séries de filmes, da internet e do mundo digital, a falta de expectativas para o futuro, os conflitos relacionados à orientação sexual e a falta de tratamento também são fatores influenciadores (CABALLERO MA, et al., 2017).

Dentre as diversas causas de pensamentos de autoextermínio nos adolescentes, vale destacar que adolescentes homossexuais apresentam ideação 3 vezes maior que outros indivíduos da mesma faixa etária. De acordo com o estudo de Santos WB e Dinis NF (2015), que conta com relatos de adolescentes que sofreram de pensamentos ou tentativas suicidas, o medo de fracassar em relações heteronormativas também pode levar a tal ato, pois a resistência exercida pelo sujeito, sem sucesso, desencadeia sofrimento e desequilíbrio. Determinados comportamentos também são associados às causas, como comportamento mais impulsivo e agressivo, principalmente se relacionado a bullying, picos de raiva, vitimização e isolamento, bem como comportamento extremamente perfeccionista (VARGAS-MEDRANO J, et al., 2020;).

Além desses fatores, adolescentes expostas ao suicídio de um membro da família, principalmente dos pais, podem causar ansiedade, depressão e a tendência, mas isso é sentido de forma mais significativa quando ocorre na infância, acarretando impactos na adolescência. O isolamento social do adolescente está associado a ideação suicida, pois se vê com falta de apoio e mentalmente vulnerável a fatores negativos. Nesses casos, os eventos estressantes da vida colaboram para a auto mutilação (KENNEBECK S e BONIN L, 2020).

\section{Fatores de risco}

Dentro do que se pode considerar fatores de risco associados ao aparecimento do comportamento suicida pode-se diferenciá-los em fatores pessoais, familiares e sociais. Os pessoais estão relacionados principalmente ao gênero, e sendo assim, destaca-se o masculino em maior proporção, com baixa expressividade emocional e com a utilização de métodos mais letais do que o gênero feminino. Há também entre os aspectos sociais o desemprego na família e baixo nível socioeconômico que geram uma escassez prolongada, e o fracasso escolar, sendo que todos esses podem ser elementos pessoais significativos para a autodestruição (SERRANO-RUIZ CP e OLAVE-CHAVES JA, 2017).

Kim KM (2019) aponta que dentre as preocupações juvenis que podem levar a ideações suicidas, a maior delas, presente em $55 \%$ dos adolescentes participantes de seu estudo, é com o desempenho escolar e a carreira, sendo essa preocupação crescente ao decorrer da adolescência, que corresponde a uma transição de fases e cobranças (PINTO LLT, et al. 2017). Seguido da preocupação escolar, vem as relações interpessoais e familiares.

Em relação aos aspectos familiares é importante ressaltar a instabilidade intrafamiliar, a pouca proximidade afetiva e histórico familiar de suicídio que são contribuintes para o isolamento do adolescente e limitação de sua vida ativa. Salienta-se ainda a violência doméstica, englobando abusos físicos e psicológicos que tem grande carga para gerar resultados como depressão e comunicação inadequada, que podem ser mediadores da ideação suicida e do suicídio consumado (PINTO LLT, et al. 2017). 
Os fatores sociais consideráveis influentes são: (I) a discriminação, que afeta a autoestima, ansiedade, induz ao abuso de álcool e pode levar à depressão; (II) a exposição a determinados conteúdos pela mídia, que pode gerar o efeito de imitação, principalmente nessa faixa etária e por indivíduos com diagnóstico psiquiátrico; e por fim, (III) a falta de redes de apoio social que reforça a vivência dos que são mais isolados e com poucos vínculos afetivos que, quando passam por conflitos, têm risco aumentado para ameaçar a própria vida (SERRANO-RUIZ CP e OLAVE-CHAVES JA, 2017).

O estudo de Faria DA (2019) mostra que adolescentes com dores crônicas apresentam o dobro de chances de morte por suicídio do que os que não sentem, sendo mais prevalente no sexo feminino, que apresentou maior percentual nas pesquisas por dor, onde $93,9 \%$ das mulheres e $80 \%$ dos homens relataram esse sintoma. As dores crônicas estão intimamente ligadas a desesperança, que por sua vez geram vulnerabilidade e comportamento suicida.

\section{Métodos utilizados para o autoextermínio}

Entre as inúmeras maneiras de tentativa de suicídio, há uma diferença dos meios utilizados de acordo com o sexo. Adolescentes do sexo feminino, apesar de tentarem de 2 a 3 vezes mais o do que o masculino, atingem menos 0 ato por usarem métodos menos agressivos. Por isso os registros de suicídio do sexo masculino são maiores proporcionalmente por conseguirem completar o feito. $O$ sexo feminino recorre mais a meios como ingestão de venenos e de medicamentos, sendo que as substâncias mais usadas são carbamato e os medicamentos psicotrópicos. Já os homens, apesar de também usarem esses métodos, recorrem de modos mais agressivos como ferimento por arma de fogo e enforcamentos (JOHNSON P, et al, 2017).

Já foi abordada na literatura a existência da dificuldade do diagnóstico dos casos de autoextermínio que chegam na urgência, mostrando a subnotificação das tentativas. Intoxicações por substâncias exógenas, por exemplo, só são notificadas como tentativa suicídio quando a família ou o indivíduo confirmam a intenção, do contrário só há o registro de intoxicação exógena (SILVA LLT, et al., 2015).

É ressaltado por autores que métodos menos graves evidenciam menos o planejamento do ato suicida, já os meios mais graves têm maior relação com o planejamento (BANDO DH e LESTER D., 2014). É possível analisar que alguns adolescentes ao realizarem a tentativa de autoextermínio se comunicam com familiares e amigos ou até utilizam lugares que outras pessoas poderiam ter fácil acesso, e assim, deixa-se notório que a intenção real não é o suicídio de fato, mas sim chamar a atenção para seu sofrimento.

Esse propósito de apenas atrair atenção de outros indivíduos, de não planejar o suicídio e apenas ingerir drogas ocorre, principalmente, quando os adolescentes estão diante de conflitos familiares ou ruptura de relacionamentos e essa tentativa é usada como uma forma de buscar mudança na realidade do lar ou da relação amorosa que vive (ARTEAGA AMP; et al., 2020). Porém deve ser considerada como tentativa de autoextermínio e um maior risco para uma segunda tentativa com sucesso, visto que muitas vezes o chamar a atenção não é levado em consideração e o sofrimento do adolescente continua a existir. Toda essa cadeia de sofrimento deve ser levada em consideração, seja a ideação, o planejamento e o ato em si da tentativa de autoextermínio (RUFINO NC, et al., 2020).

\section{As Escalas de Beck}

Adolescentes possuem maior propensão a pensamentos suicidas, principalmente, diante de uma perturbação ou causa de grande importância para ele. Esses pensamentos podem ser frequentes e por muito tempo e, dessa forma, podem desencadear o comportamento suicida. A ideação do suicídio está ligada ao maior risco de futura tentativa de autodestruição; já o planejamento suicida relaciona-se com um grande risco de morte. Para identificar a ideação suicida uma das escalas utilizadas é a Escala de Ideação Suicida de Beck (AMARAL AP, et al., 2020).

A Escala que possui vinte e um itens, dentre eles dezenove itens com três respostas alternativas que demonstram a progressividade da gravidade dos desejos e planos, e abordam as seguintes perguntas: Desejo de viver; Desejo de morrer; Razões para viver ou morrer; Tentativa de suicídio; Tentativa de suicídio passiva; 
Duração das ideias de suicídio; Frequência da ideação; Atitude em relação à ideação; Controle sobre atos suicidas; Inibições para a tentativa; Razões para a tentativa; Especificidade do planejamento; Acessibilidade ou oportunidade do método; Capacidade de realizar a tentativa; Probabilidade de tentativa real; Extensão da preparação verdadeira; Bilhete suicida; Atos finais; Despistamento segredo. E 2 itens são informativos relacionados com número de tentativas prévias de suicídio e com a seriedade do intuito de morrer na última das tentativas (BANDO DH e LESTER D; 2014)

Há também o Inventário de Depressão de Beck que auxilia na análise dos sintomas da depressão. A literatura sugere sua aplicação juntamente com a Escala de Ideação Suicida. O Inventário de Depressão possui 21 itens de auto relato, com quatro opções de respostas (de 0 a 3) também demonstrando a escala de gravidade, mas agora da depressão. Existe ainda a Escala de Desesperança que tem 20 itens com afirmações, com respostas de certo ou errado e analisa as expectativas negativas acerca do futuro. As escalas e inventários de Beck são utilizados como instrumentos de avaliação para investigação de sintomas depressivos e ideação suicida (AMARAL AP, et al., 2020).

\section{Estratégias de prevenção}

De acordo com a literatura, os fatores de risco, em companhia das estratégias de prevenção, caminham juntos e são classificados como um modelo de comportamento suicida, ressaltando a oposição dos fatores biológicos, como genética, fatores cognitivos e emocionais, e ambientais, como situações familiares e sociais adversas, cultura, espiritualidade e trajetória de vida (CONDORELLI R, 2016). Por isso, as estratégias de prevenção devem basear-se no contexto ambiental do indivíduo, avaliando os fatores de risco para que se tenha uma estratégia de prevenção competente, pois ela só pode ser considerada como tal quando é capaz de amenizar o fator de risco associado (RUFINO NC, et al., 2020).

Dessa forma, diferentes métodos são aplicados para o rastreio da ideação suicida. De forma geral, uma maneira para avaliar um adolescente é indagando sobre a ideação suicida diretamente, simplesmente perguntar sobre fatores de risco, pensamentos suicidas e intenções. A preocupação e medo de que falar, ou perguntar, sobre o suicídio irá provocar concepções ou ações suicidas em uma criança ou adolescente não é sustentada por evidências. Após avaliar o risco, pode ser necessário o encaminhamento para a equipe psiquiátrica, para uma melhor avaliação da saúde mental, considerando os riscos. Portanto, quando estes adolescentes estão em uma crise aguda devido a pensamentos de autoextermínio, o foco da intervenção é mantê-los seguros até que o risco cesse ou diminua. Na maioria dos casos é necessário auxílio familiar ou de outros indivíduos que estejam dispostos a apoiar e permanecer com o paciente durante toda a crise (KENNEBECK S e BONIN L, 2020).

Além disso, as opções de tratamento podem incluir hospitalização, medicamentos para transtornos psiquiátricos subjacentes, como depressão maior, intervenção psicológica mais frequente. É importante salientar, que quando analisadas as tentativas de suicídio e seus fatores de risco/prevenção é recomendado psicoterapia para que seja tratada a depressão e sejam produzidas estratégias de enfrentamento. Sendo assim, a terapia intensiva pode ser uma ótima opção para estabilização de uma crise, com notáveis resultados a curto, médio e longo prazo (KENNEBECK S e BONIN L, 2020). O tratamento medicamentoso e não medicamentoso, para os transtornos de humor, ou transtornos diagnosticados, devem ser iniciados o mais breve possível (SHAIN B, 2016).

Delimitar os jornais, revistas, programas de televisão ou qualquer outro meio de comunicação que venha a divulgar notícias de cunho suicida pode ser necessário, principalmente avaliando a forma como a notícia é descrita, pois o adolescente apresenta mais tendência que o adulto para imitar o comportamento propagado por essas mídias. Ademais, monitorar o tempo gasto em mídias sociais é importante, pois exceder 5 horas diárias está intimamente associado a altos níveis de depressão e suicídio. Todavia, a internet não é de um todo ruim, existem fóruns de debate que apresentam impactos psicológicos positivos, uma vez que se trata de um espaço livre de rótulos onde os adolescentes se sentem livres para conversar e apoiar uns aos outros, sendo a educação em saúde e a informação sobre como buscar ajuda quando se está em sofrimento mental uma boa forma de prevenção da tentativa de autoextermínio e automutilações corporais sem a intenção de se matar (SHAIN B, 2016). 


\section{CONSIDERAÇÕES FINAIS}

As evidências demonstram um aumento expressivo do número de casos de suicídio entre os jovens. A maior parte deles possui algum transtorno psíquico associado que pode ser reforçado por fenômenos como instabilidade familiar, discordâncias socioculturais, problemas de gênero, abuso de álcool e drogas. Nesse sentido, é notório que a fase da adolescência é um momento de grandes transformações no corpo e na psique do jovem, por isso, é necessário que profissionais da saúde, familiares e amigos estejam atentos às mudanças comportamentais durante esse período. Verificou-se, ainda, que é imprescindível indagar diretamente ao jovem acerca da ideação suicida, uma vez que a censura social a respeito do suicídio pode corroborar para ocultar o sofrimento do jovem e continuar fomentando diversos tabus relacionados a essa problemática. Além disso, vale salientar que existem estratégias de prevenção e opções de tratamento que são cruciais no que diz respeito à diminuição dos casos de suicídio e do sofrimento do adolescente.

\section{REFERÊNCIAS}

1. BANDO DH, LESTER D. An ecological study on suicide and homicide in Brazil. Cien Saude Colet 2014; 9(4): 11791189.

2. BIRMAN J. Mal-Estar na atualidade, a psicanálise e as novas formas de subjetivação. Rio de Janeiro.Editora: José Olympio. 2016.

3. CABALLERO MA, et al. Principales factores de riesgo relacionados con el intento suicida en un grupo de adolescentes. Revista Médica de Santiago de Cuba - Medisan, 2017.

4. CIDADE NOP, ZORNIG SMAJ. Automutilações na adolescência: reflexões sobre o corpo e o tempo. Estilos da Clínica, 2021.

5. CONDORELLI R. Social complexity, modernity and suicide: an assessment of Durkheim's from the perspective of a non-linear analysis of complex social systems. Springerplus 2016; 5(374): 1-56.

6. CONSELHO FEDERAL DE PSICOLOGIA: SAF/SUL. Suicídio e os desafios para a Psicologia. Dec. 2017. Disponível em: https://site.cfp.org.br/publicacao/suicidio-e-os-desafios-para-a-psicologia/

7. DURKHEIM E. O suicídio: estudo de sociologia. São Paulo: WMF Martins Fontes; 2011.

8. FARIA DA, et al. Physical pain and hopelessness in school teenagers. BrJP, São Paulo; 2020.

9. JAEN-VARAS D, et al. A associação entre taxas de suicídio de adolescentes e indicadores socioeconômicos no Brasil: um estudo ecológico retrospectivo de 10 anos. Revista Brasileira de Psiquiatria, setembro-outubro, 2019.

10. JOHNSON P, et al. Legal origin and social solidarity: the continued relevance of Durkheim to comparative institutional analysis. Sociology 2017; 51(3): 646-665.

11. KENNEBECK S, BONIN L. Ideação e comportamento suicida em crianças e adolescentes: avaliação e gestão. UpToDate, 2020.

12. KIM KM. What makes adolescents psychologically distressed? Life events as risk factors for depression and suicide. Eur Child Adolesc Psychiatry, 2020.

13. MINISTÉRIO DA SAÚDE. Secretaria de Vigilância em Saúde. Boletim Epidemiológico v. 50, nº 24: Perfil epidemiológico dos casos notificados de violência autoprovocada e óbitos por suicídio entre jovens de 15 a 29 anos no Brasil, 2011 a 2018. Brasília - DF, Brasil, 2019. Disponível em: https://antigo.saude.gov.br/images/pdf/2019/setembro/13/BE-suic--dio-24-final.pdf

14. OLIVEIRA JWT, et al. Características das tentativas de suicídio atendidas pelo serviço de emergência pré-hospitalar: um estudo epidemiológico de corte transversal. J. bras. psiquiatr., Rio de Janeiro, 2020.

15. PINTO LLT, et al. Tendência de mortalidade por lesões autoprovocadas intencionalmente no Brasil no período de 2004 a 2014. Jornal Brasileiro de Psiquiatria 2017; 66(4): 203-210

16. RIBEIRO JM, MOREIRA MR. Uma abordagem sobre o suicídio de adolescentes e jovens no Brasil. Ciência \& Saúde Coletiva [online]. 2018; 23(9): 2821-2834.

17. RUFINO NC, et al. Suicide Attempts Among French and Brazilian Adolescents Admitted to an Emergency Room. A Comparative Study of Risk and Protective Factors. Front. Psychiatry 11:742. 2020.

18. SANTOS WB, DINIS NF. Violência e risco de suicídio na construção das masculinidades adolescentes. Cad. Pagu, Campinas, 2018.

19. SERRANO-RUIZ CP, OLAVE-CHAVES JA. Artículo de investigación científica y tecnológica / Revisión. Factores de riesgo asociados con la aparición de conductas suicidas en adolescentes, Med UNAB, 2017; 20(2):139-147.

20. SILVA LLT, et al. O suicídio na adolescência nas publicações da enfermagem brasileira: revisão integrativa da literatura. Revista de Enfermagem do Centro Oeste Mineiro, 2015.

21. TSAI AC, et al. From social networks to health: Durkheim after the turn of the millennium. Soc Sci Med 2015; 125: 17.

22. VARGAS-MEDRANO J, et al. Psychological and neurobiological aspects of suicide in adolescents: Current outlooks. Department of Psychiatry, UT Southwestern Medical Center at Dallas Dallas, 2020. 